\title{
Corela
}

Cognition, représentation, langage

16-2 | 2018

Vol. $16, n^{\circ} 2$

\section{Discours aux Nations Unies : des normes pour un genre « poli »?}

Olivia De Mattei Tchemako

\section{(2) OpenEdition}

\section{Journals}

\section{Electronic version}

URL: http://journals.openedition.org/corela/6956

DOI: $10.4000 /$ corela.6956

ISSN: 1638-573X

\section{Publisher}

Cercle linguistique du Centre et de l'Ouest - CerLICO

\section{Electronic reference}

Olivia De Mattei Tchemako, «Discours aux Nations Unies : des normes pour un genre «poli »? », Corela [Online], 16-2 | 2018, Online since 07 December 2018, connection on 30 April 2019. URL : http:// journals.openedition.org/corela/6956; DOI : 10.4000/corela.6956

This text was automatically generated on 30 April 2019.

\section{(c) (i) (2)(2)}

Corela - cognition, représentation, langage est mis à disposition selon les termes de la licence Creative Commons Attribution - Pas d'Utilisation Commerciale - Partage dans les Mêmes Conditions 4.0 International. 


\title{
Discours aux Nations Unies : des normes pour un genre « poli »?
}

\author{
Olivia De Mattei Tchemako
}

\section{Introduction}

1 Chaque année au siège des Nations Unies, un débat général réunit les chefs d'État et de gouvernement $\mathrm{du}$ monde pour des discussions diplomatiques de haut niveau qui amorcent la session annuelle de l'Assemblée générale. Il s'agit d'un événement médiatisé durant lequel les représentants des États membres prononcent à tour de rôle des discours qui abordent des sujets d'actualité et des problématiques d'ordre mondial, qui donnent une orientation et une impulsion aux travaux de l'Assemblée. Ces discours marquent une circonstance unique dans l'année, la rencontre onusienne incontestablement la plus importante, $d u$ point de vue du nombre des États membres participants et du caractère éminent des sujets traités. C'est donc également l'occasion de renforcer les valeurs sur lesquelles se fonde l'Organisation, et réactiver les idéaux incarnés par la Charte. À cette occasion d'ailleurs, des requêtes sont régulièrement formulées et reflètent les préoccupations des État-membres. C'est ainsi que l'on voit l'importance accordée aux questions telles que l'élargissement du Conseil de sécurité, le droit de véto, les conflits régionaux, le nucléaire, la lutte contre la pauvreté, les embargos, le statut de Taïwan, les réformes globales de l'Organisation, les changements climatiques, les aides au développement, pour ne citer que celles-là.

\section{Problématique, corpus et méthode}

Cette contribution entame un travail de caractérisation de ce sous-genre diplomatique, auquel appartiennent des discours prononcés par des hommes politiques dans une enceinte institutionnelle. Elle étudie précisément les manifestations de la politesse diplomatique à travers la formulation des actes de requête; et entend montrer l'impact d'un contexte socio-discursif, des contraintes qui en découlent, sur le fonctionnement de 
la langue et le sens des formes linguistiques. Le corpus d'étude se compose de discours émanant de pays francophones et prononcés en français lors des débats de l'Assemblée Générale. Il s'agit d'un corpus contrastif qui compte les discours prononcés par des représentants d'Europe occidentale et d'Afrique centrale sur une quinzaine d'années (2000-2014); et qui exploite les différences entre des pays ayant des situations bien différentes, pour une étude comparative. Ce sont la France, la Belgique, la Suisse, le Luxembourg, le Cameroun, le Gabon, la République Centrafricaine, le République du Congo, la République Démocratique du Congo et le Tchad. L'on a ainsi des pays jeunes et des pays ayant une longue histoire nationale, des anciennes colonies et des anciennes métropoles, des pays développés et des pays en voie de développement, des pays ayant des frontières communes mais un poids et une situation géopolitique bien différents.

3 La méthode d'étude quali-quantitative allie les outils d'Analyse du discours à ceux de la Logométrie; ces derniers se complétant les uns les autres. Une base de données contrastive séparée en dix parties correspondant aux différents pays a donc été créée à partir du corpus, pour un traitement automatique par un logiciel de Logométrie. D'une part, une approche inductive consiste à procéder à des requêtes non focalisées sur ledit logiciel, afin de faire émerger des informations, des questionnements qui à leur tour seront approfondis à l'aide des outils d'Analyse du Discours. C'est ainsi que des recherches générales sur les spécificités lexicales, la distribution des codes grammaticaux, les distances lexicales et grammaticales entre les textes, les segments répétés, les mots et phrases-clés mènent à des résultats dont l'étude en détail se fait à l'aune de l'AD. Inversement, une approche hypothético-déductive permet d'examiner de près les discours à l'aide d'outils et de faits de langue jugés pertinent pour étudier un genre en linguistique. À cet effet, l'on s'intéresse à l'appareil de l'énonciation, aux actes de langage et l'efficacité du discours, aux procédés de désignation, à l'implicite... La contribution de la Logométrie dans cette deuxième approche se veut irrécusable dans la mesure où, vue l'étendue du corpus (159 discours), une aide logicielle est indispensable pour pouvoir repérer, à l'aide de requêtes documentaires, focalisées cette fois, les éléments de discours précisément recherchés (pronoms, substantifs, modaux...).

4 Pour amorcer l'exploration du corpus et en avoir une vision d'ensemble, un calcul des spécificités a été mené. Il s'agit d'un outil permettant de repérer le vocabulaire objectivement spécifique d'une partie du corpus par rapport à l'ensemble du corpus. Ici, ce qui rend un terme spécifique à un locuteur est que son utilisation par ce locuteur tranche par son importance par rapport à la moyenne générale du corpus. Autrement dit, par rapport à l'utilisation moyenne dudit terme dans la totalité du corpus, le locuteur le surutilise ${ }^{1}$.

5 Le tableau suivant regroupe par ordre d'importance les premiers résultats du calcul des spécificités, que nous limitons pour cette étude aux noms et aux adjectifs, considérant que ces derniers peuvent donner une idée des thématiques auxquelles s'intéressent les États dans leurs discours ${ }^{2}$. Ce tableau montre donc les noms et adjectifs (ramenés en lemmes) apparaissant en plus haute spécificité positive, et qui partant caractérisent le mieux le discours de chacun des pays par rapport aux autres. 
Les 12 noms et adjectifs -ramenés à leur forme canonique- les plus surutilisés

Par ordre d'importance:

\begin{tabular}{|l|l|l|l|l|l|l|l|l|l|l|}
\hline Belgique & France & Luxembourg Suisse & Cameroun & Congo & Gabon & RCA & RDC & Tchad \\
\hline Belgique & France & Luxembourg & Suisse & Cameroun & Congo & Gabon & Rép.centraf & Congo & Tchad \\
\hline collègue & Europe & luxembourgeois & Onu & paul biya & central (Afr) & gabonais & centrafricain & démocratique & tchadien \\
\hline cher & européen & européen & droit & Camerounais & Brazzaville & Bongo & délégation & république & honorable \\
\hline européen & monde & matière & Genève & OMD & Afrique & effort & Séléka & congolais & réfugié \\
\hline belge & union & kosovo & suisse & différend & africain & VIH & transition & Kivu & Idriss \\
\hline responsabilité peuple & dossier & humanitaire golfe & sous-région & Afrique & François & territoire & Deby \\
\hline citoyen & inde & responsabilité & humain & excellence & forestier & Sida & Bozize & Monuc & soudanais \\
\hline droit & diversité & action & neutralitén & DSCE & bassin & session & Centrafricain & FDLR & Darfour \\
\hline discrimination seul & multilatéralisme homme & Bakassi & comité & remboursement Cemac & rwandais & Taiwan \\
\hline essentiel & spéculation brut & G20 & maritime & Denis & dette & Bangui & infrastructure & frère \\
\hline prospérité & taxe & chiffre & jeune & Camerounais & réunion & développement agricole & ordinaire & délégué \\
\hline facile & palestinien & contexte & divergence & inévitable & forêt & Ondimba & centrafrique & réunification & lac Tchad \\
\hline
\end{tabular}

6 Pour tous les pays représentés, l'analyse des spécificités fait apparaître les noms desdits pays en plus haute spécificité positive. Cela signifie que, pour chacun des 10 ensembles de discours sans exception, le nom du pays représenté par celui qui prend la parole est le mot le plus surutilisé. Cela signifie également que cet attribut, ce trait saillant qui distingue le mieux les prises de parole est la caractéristique majeure de chaque discours suivant le pays dont il émane. L'on peut de prime abord expliquer la proéminence de cette spécificité précise par le fait que chacun des locuteurs parle depuis son propre point de vue. Cependant, on constate que dans ces discours où les États membres sont à l'honneur, il est usuel que ceux-ci prennent eux-mêmes la parole et parlent en leur propre nom. Concrètement, les représentants des États emploient très souvent la troisième personne et font parler leur pays. Ainsi, les noms de pays remplacent le Nous, ont la fonction de sujet (syntaxique et sémantique) et prennent en charge l'énonciation. C'est le cas dans :

(1) La France ne cesse de demander, depuis 1982, la création d'un État palestinien. Ou encore :

(2) Le Gabon appelle à la destruction de tous les arsenaux chimiques dans le monde.

8 Ces pays deviennent donc des locuteurs et accomplissent par là même des Actes de langage ${ }^{3}$.

9 Parmi ces actes, ceux qui ont suscité notre intérêt sont les actes directifs, avec leur formulation souvent indirecte qui révèle très certainement une caractéristique importante du genre. C'est sur ce constat que nous allons relever les différentes formes d'indirections, sachant que celles-ci participent à la mise en place de la politesse dans les discours diplomatiques. Le cadre théorique est défini par les outils de l'analyse pragmatique, ainsi que les acquis de l'analyse du discours. À ce propos, dans son ouvrage Analyser les discours institutionnels (2013), Alice Krieg-Planque mentionne que « Dans bien des cas, la politesse est l'un des éléments qui expliquent le recours à des actes de langage indirects » (p. 65) . 


\section{Actes de Langage indirects: Enjeux de la caractérisation d'un genre}

10 Rappel: Les actes de langage correspondent aux différentes actions que l'on peut accomplir par des moyens langagiers.

11 La paternité de leur théorie est attribuée à John Austin ${ }^{5}$; mais la taxinomie des actes retenue par nos travaux est celle de John Searle. Ainsi, «nous disons à autrui comment sont les choses, nous essayons de faire faire des choses à autrui, nous nous engageons à faire des choses, nous exprimons nos sentiments et nos attitudes et nous provoquons des changements par nos énonciations » (Searle, 1982, p.70). Ces différentes actions, qui sont concrétisées par des verbes illocutoires (verbes par lesquels sont réalisés les actes de langage), correspondent respectivement selon Searle à des catégories de base qui sont : les actes assertifs, directifs, promissifs, expressifs et déclaratifs ${ }^{6}$.

Les actes de langage peuvent se réaliser directement, mais très régulièrement dans le corpus, on rencontre des formulations indirectes, dans lesquelles un acte emprunte les apparences d'un autre. Il est question pour le locuteur de parler afin d'informer mais aussi afin de susciter une conduite dont il laisse le choix et le jugement aux auditeurs. C'est le cas dans :

(3) La République Centrafricaine reste favorable à ce que l'Organisation des Nations Unies procède à une réforme nécessaire et rationnelle suivant l'évolution de la situation internationale (...) qu'il s'agisse de la revitalisation de l'Assemblée Générale et de la nécessaire réforme du Conseil de sécurité (...).

Et aussi dans :

(4) La République Démocratique du Congo fait observer l'impérieuse nécessité de l'organisation et de la tenue d'une conférence internationale sur le financement de sa reconstruction, et ce, dans les meilleurs délais.

Il s'agit d'actes de langage indirects, expression elliptique pour «actes de langage formulés indirectement, sous le couvert d'autres actes de langage ».D'après Searle, accomplir un acte indirect consiste généralement à affirmer, ou questionner sur l'une des conditions de réussite auxquelles est soumis l'acte en question, c'est-à-dire effectuer un déplacement, de l'acte lui-même à l'une ou l'autre de ses conditions de "félicité»". Dans ces deux cas, l'acte directif se fait passer pour un assertif, dans lequel on pose la condition de sincérité portant sur le locuteur (précisément l'État-membre locuteur). Si l'on considère par exemple que la République Démocratique du Congo est en train de demander indirectement la tenue effective d'une conférence portant sur le financement de sa reconstruction, elle montre sa sincérité en en faisant observer l'impérieuse nécessité. Les représentants décrivent leur pays en train d'avoir une attitude qui est censée à son tour suggérer logiquement une réaction favorable concrète et pratique de la part des interlocuteurs (même si elle est souvent différée). Ici, en réalité, c'est l'usage de la troisième personne qui donne l'occasion de construire la requête de manière indirecte. À la place du je ou du nous, ou plus directement de l'impératif, l'on parait faire une description d'une entité à laquelle on s'identifie mais qui est tout de même autre que soimême (le représentant dit que la République Centrafricaine reste favorable...). À cause de l'usage de cette troisième personne, la formulation indirecte devient presque automatique. 

en priorité aux États-membres que la parole est donnée. C'est pourquoi dans les discours, l'État se superpose à la parole de la personne même du représentant et parle en son propre nom. L'État-membre devient LE locuteur, et ce qui est formulé comme une description ou un constat de la part dudit représentant s'actualise donc en requête (même indirecte), étant donné que l'émetteur de la requête est bien celui qui prend la parole. L'énoncé «La République Centrafricaine reste favorable... à une réforme nécessaire et rationnelle » ne décrit pas simplement une attitude de la RCA ; c'est la RCA elle-même qui demande indirectement la réforme.

Soient les énoncés :

(5) Pour sa part, la République Centrafricaine souhaite que lors de la conférence de Bali en décembre prochain, les pays nantis honorent enfin leurs engagements dans le domaine du transfert de technologies et apportent une assistance... pour une lutte efficace contre le changement climatique.

(6) C'est pour cela que le Tchad souhaite que les États Unis d'Amérique mettent un terme rapidement à cet embargo (cubain) qui est contraire à leur nouvelle vision du monde et contraire aux grands principes que défend notre organisation.

(7) La France quant à elle souhaite deux évolutions. Inverser, tout d'abord, la tendance à l'augmentation des contributions volontaires au détriment des contributions obligatoires. Avancer, en second lieu, dans la mobilisation de ressources pour le développement et pour l'aide au développement.

(8) La France souhaite que le retrait de Gaza en soit une première étape (au processus de paix agréé par le conseil de sécurité).

(9) La convention qui bannit ces armes (mines antipersonnel) est entrée en vigueur en 1999 et la Belgique espère qu'elle sera renforcée lors de la conférence de révision...à Carthagène, en Colombie.

(10) La Belgique souhaiterait qu'une relation directe entre le Président du Congo et le Président du Rwanda puisse s'établir et contribue à accélérer l'aboutissement d'une solution.

(11) Le Cameroun... souhaite une mise en place rapide de la commission de consolidation de la paix et du conseil des droits de l'homme ...

(12) La France souhaite des engagements plus forts concernant notamment les transferts de technologies en faveur des pays émergents.

Ces énoncés se présentent de prime abord sous forme de simples assertions, mais il s'agit en réalité d'actes directifs indirects. En effet, il n'y a aucun usage du mode impératif, mais les différents pays-locuteurs demandent de réaliser leurs souhaits bien plus qu'ils ne renseignent simplement sur leurs aspirations. On parle respectivement ici de valeur dérivée et valeur littérale de l'acte. Cette forme d'indirection est dite conventionnelle, étant donné que tout le monde peut s'accorder sur le fait qu'en langue française, les

Corela, $16-2$ | 2018 
verbes exprimant le souhait ou la volonté tels que vouloir, souhaiter, aimer, désirer, aspirer, tenir à... peuvent introduire une requête. L'indirection conventionnelle repose dans ces exemples (et dans le corpus en général) sur l'affirmation du désir ; même si cette forme d'indirection peut employer d'autres moyens tel que le questionnement sur la capacité, la volonté ou la possibilité pour l'interlocuteur de réaliser le souhait émis. L'on aurait alors, pour le deuxième exemple sur le Tchad s'adressant aux États-Unis (exemple 6): Pouvezvous (ou pourriez-vous) mettre rapidement un terme à cet embargo qui est contraire à la nouvelle vision du monde... ? Ou encore pour la RCA (exemple 5) lorsqu'elle s'adresse à ceux qu'elle nomme "pays nantis »: Voulez-vous enfin honorer vos engagements ... et apporter une assistance...? Mais ces autres moyens, parce qu'ils paraissent dans ce contexte trop injonctif et donnent à voir une hostilité manifeste, ne correspondent certainement pas au genre de discours étudié 8 . C'est pourquoi on n'en retrouve aucune occurrence dans le corpus.

Ainsi, on parle de tournures self-oriented pour celles qui portent sur l'affirmation du désir; en opposition aux other-oriented qui mettent en avant le destinataire. Tournures qui sont toutes - on l'aura compris - contraires à toute formulation directe de requête, qui voudrait que l'on use du mode impératif. De ce fait, la RCA par exemple "souhaite " que les pays nantis honorent leurs engagements et apportent une assistance aux pays sous-développés, mais elle ne les interroge pas sur leur capacité ou leur volonté de le faire; et elle leur en intime encore moins l'ordre. Elle dit simplement le souhaiter, et cela vaut requête.

\section{Des faits de langue qui complètent l'indirection :}

Plusieurs des énoncés précités recourent à la voix passive, la voix pronominale et la nominalisation, ce qui favorise l'effacement des actants auxquels s'adressent les requêtes. C'est la formulation indirecte des actes (troisième personne suivie du verbe de volonté) qui rend ces procédés possibles ${ }^{9}$. Ainsi, lorsque la France dit souhaiter «le retrait» de Gaza dans le cadre du processus de paix, elle ne mentionne pas en discours l'identité de celui qui doit se retirer ${ }^{10}$. De même, qui est-ce qui doit apporter des contributions obligatoires et mobiliser des ressources pour l'Aide Publique au Développement? Ou encore s'engager plus sérieusement sur les transferts de technologies vers les pays émergents? L'on y répondra tout de suite qu'il ne sert à rien de nommer les concernés puisque tout le monde sait plus ou moins de qui il s'agit; et que tout cela pourrait tout simplement correspondre à un style qui s'applique au genre de discours étudié. Ou bien peut-être qu'au contraire, ce serait bien sciemment que les pays, dans une tentative plus ou moins fructueuse de sembler moins coercitifs afin d'éviter toute forme de confrontation possible, s'empêcheraient autant que faire se peut de se désigner directement. Prenons le cas du sixième exemple, pour la voix pronominale, sur la Belgique qui «souhaiterait qu'une relation directe entre le Président du Congo et le Président du Rwanda puisse s'établir et contribue à accélérer l'aboutissement d'une solution ». Ici, les deux actants sont bien cités; sauf que grâce à la voix pronominale, ce qui est mis en avant c'est la relation qui peut s'établir entre eux, par elle-même donc. Sachant pertinemment qu'en réalité une relation ne saurait s'établir elle-même, et que les deux personnes citées sont seules à pouvoir la créer, la Belgique aurait pu dire autrement (à la voix active) qu'elle souhaite que les présidents congolais et rwandais établissent une relation directe. Mais le dire ainsi donnerait peut-être à voir que la Belgique dit aux concernés ce qu'ils ont à faire. Il parait alors moins coercitif, moins injonctif de le 
formuler à la voix pronominale. L'influence de ce type de procédés sur l'efficacité des actes de langage pourrait faire l'objet d'une étude spécifique. Emmanuelle Danblon (2010) montrait déjà dans son article intitulé «À quelles conditions une charte est-elle efficace ?» que la voix passive à elle seule suffit à biaiser la légitimité d'une déclaration officielle institutionnelle ${ }^{11}$.

En résumé, ce type de formulation (voix passives et pronominales, nominalisations) répond-il simplement à une contrainte de style que le genre impose, ou bien doit-on se résoudre à y voir une tentative expresse de ménager ceux à qui on s'adresse? Les deux propositions peuvent aussi se compléter, si l'on postule qu'il s'agit d'un genre dont les exigences sont mises au service de la volonté globale d'écarter -au moins en discours-les sources potentielles de confrontation, et de valoriser le consensus ${ }^{12}$. Ces exigences définissent alors les normes qui s'appliquent au genre, concrétisées par des procédés qui favorisent l'élimination des actants.

Dans tous les cas, si c'est la formulation indirecte qui autorise les procédés de nominalisation et de voix passive et active, ceux-ci l'accentuent et la consolident.

En définitive, les requêtes indirectes conventionnelles sont construites par les pays comme suit :

L'État-membre (une entité autre que la personne même du représentant, mais à qui ce dernier s'identifie quand-même) $\rightarrow$ désire $\rightarrow$ que l'assistance réalise quelque chose

La RCA $\rightarrow$ souhaite $\rightarrow$ que les pays nantis honorent enfin leurs engagements

Ou plus indirectement encore :

L'État-membre (une entité autre que la personne même du représentant, mais à qui ce dernier s'identifie quand-même) $\rightarrow$ désire $\rightarrow$ que quelque chose se réalise/la réalisation de quelque chose (qui est à la portée de l'assistance)

La Belgique/La France $\rightarrow$ souhaite $\rightarrow$ qu'une relation puisse s'établir / des engagements plus forts concernant le transfert des technologies en faveur des pays émergents

\section{b. Des requêtes indirectes non conventionnelles}

En dehors des directifs indirects conventionnels, l'on retrouve des indirects non conventionnels dans le corpus.

Soit la liste d'énoncés suivante :

(13) Et c'est fort de cette approche que nous nous préparons à participer à la Conférence Internationale sur la paix, la sécurité, la démocratie et le développement dans la région des Grands-Lacs. Nous entendons saisir cette chance historique pour conjurer les démons de la haine et de la violence et jeter les bases d'une coexistence pacifique entre nos peuples et nations qui ont un destin en commun. En réaffirmant la centralité de la République Démocratique du Congo dans cette conférence, je voudrais relever que la République du Congo, si proche de la RDC à maints égards, comprendrait mal qu'elle assiste en observateur à cette échéance capitale; et il en serait de même en tout point de vue, pour d'autres pays voisins de la RDC tels l'Angola et la République Centrafricaine.

(14) Le Conseil de Sécurité joue lui aussi un rôle important dans la prévention de conflits. La Suisse saluerait un engagement plus fort et plus durable du Conseil de Sécurité dans la diplomatie préventive. Pour pouvoir apporter une contribution efficace à la paix et à la sécurité, le Conseil de Sécurité doit s'adapter aux nouvelles réalités et refléter les nouveaux rapports de force politiques du $21^{\mathrm{e}}$ siècle. 
(15) La situation au Myanmar est préoccupante. La Suisse privilégie le dialogue entre toutes les parties impliquées sous l'égide du Conseiller spécial du Secrétaire Général des Nations Unies.

(16) La Suisse est persuadée que la définition des Objectifs du Développement Durable contribue largement à renforcer ce développement. Elle participera activement à la définition de cet ensemble d'objectifs. La Suisse juge impératif de regrouper dans un même processus les discussions sur l'Agenda post 2015 et sur les Objectifs du Développement Durable. évidents, qui mènerait à reconnaître une requête même indirecte, tel que les verbes de volonté qui apparaissent dans les actes indirects conventionnels. C'est l'interprétation et le dispositif énonciatif ${ }^{13}$ qui permettent de dire qu'il s'agit de requêtes. La République du Congo par exemple ne pose pas l'impératif d'être plus qu'un observateur lors de la conférence sur la paix dans les Grands Lacs; elle ne dit pas non plus simplement le souhaiter comme nous l'avons vu avant ; mais elle évoque le mécontentement qui serait le sien au cas où elle ne jouerait pas un rôle plus important dans ce qu'elle considère comme une échéance historique qui la concerne de près, ainsi que d'autres pays voisins. Il appartient ici aux interlocuteurs concernés d'entendre cela comme une demande indirecte; qui n'est cependant pas formulée comme les normes de la langue l'envisagent en général. Il s'agit d'une requête indirecte non-conventionnelle. C'est vraisemblablement le même procédé que la Suisse utilise en évoquant l'accueil qu'elle réserverait à un engagement plus ferme du Conseil de Sécurité dans la diplomatie préventive (elle le saluerait). L'énoncé suivant utilise le même procédé d'indirection :

(17) Membre du groupe encore trop sélect du G7 - avec une APD qui a atteint en 2007 près de $0,9 \%$ du RNB en attendant d'atteindre l'objectif d'un pourcent que nous nous sommes fixés- le Luxembourg ne pourrait que se réjouir d'un élargissement rapide de ce club.

Avec une approche différente cette fois, lorsque la Suisse dit "privilégier le dialogue » entre les parties au conflit au Myanmar, le sens littéral de l'énoncé donne à voir une simple opinion qui est donnée, tandis que le sens dérivé porterait sur une recommandation ; l'un étant la condition de sincérité de l'autre. Ainsi, pour que la Suisse recommande le dialogue entre les parties, il faudrait que ce soit la solution qu'elle préfère; et inversement, c'est parce qu'elle dit privilégier le dialogue qu'on peut en déduire qu'elle le recommande. De même, si dans le dernier exemple, la Suisse «juge impératif » de regrouper dans un même processus les négociations sur l'Agenda 2015 et les ODD, cela se traduit en d'autres termes par le fait de penser qu'on doit les « regrouper dans un même processus ", et que, certainement, elle appelle l'assistance à le faire.

L'exemple suivant montre une autre façon dont peuvent se construire les requêtes indirectes non conventionnelles :

(18) Il ne sert à rien de se scandaliser de la thèse du choc des civilisations ou de la nier : il faut de toutes nos forces lutter contre un tel risque, qui n'est pas totalement imaginaire, et contre ceux qui espèrent en faire une réalité. Ce devrait être d'abord pour nous tous une impérieuse raison supplémentaire pour trouver une solution aux crises régionales avant tout au Proche-Orient: La France ne cesse de demander, depuis 1982, la création d'un État palestinien. Naturellement celui-ci devra être viable, démocratique et pacifique.

Selon la France, faire barrage à la thèse du choc des civilisations constitue une raison importante de résoudre les crises régionales, et en priorité au Proche-Orient. Dans cette même lancée, elle ne demande pas de façon directe la création d'un État palestinien, mais 
elle rappelle qu'elle n'a cessé de le faire depuis 1982. Le rappel d'une demande qui n'a pas été satisfaite peut s'interpréter ici comme la réitération de la demande. La suite de l'extrait conforte cela, lorsque la France précise les conditions dans lesquelles l'État palestinien «devra naturellement " être dans le futur, si, enfin, «création d'un État palestinien " il y a. Par ailleurs, une forme de reproche pourrait doubler cette requête indirecte. Après tout, s'il relève des compétences de l'Onu de voter la reconnaissance de la Palestine en tant qu'État en son sein, si la France ne cesse de demander parce qu'elle ne l'a toujours pas obtenu, c'est vraisemblablement une déception, une frustration pour celle qui estime qu'une entité légitime jouit des moyens et capacités de répondre à des attentes raisonnables mais ne le fait pas. Les conditions seraient donc réunies pour construire -à tort ou à raison- un acte de reproche ${ }^{14}$. notre cas) que les normes de la langue permettent d'identifier comme porteurs de requêtes; tandis que la valeur de requête des seconds se saisit par interprétation. Cependant, le problème de l'interprétation c'est qu'elle présente en général des risques liés à son aspect aléatoire. Il doit y avoir une multitude de moyens d'exprimer une requête de manière non-conventionnelle. Dans les exemples cités, certains locuteurs décrivent l'attitude qui serait la leur si un évènement donné survenait ou non (expressif au conditionnel) ; d'autres informent sur leur préférence au sujet d'une situation donnée ; d'autres encore font savoir ce qui leur parait nécessaire, obligatoire, impérieux; et d'autres enfin rappellent simplement une demande insatisfaite depuis tant d'années. En bref, toutes ces méthodes n'ont rien en commun, et il n'y a pas réellement de règle de langue qui prouve incontestablement qu'elles induisent des requêtes. Dans bien des contextes, cette contrainte d'interprétation donnerait la possibilité à un interlocuteur de mauvaise foi de décider de prendre ce qui est dit aux mots, et de tout bonnement se refuser de l'interpréter, sans être accusé d'altérer les codes de la langue. À une tournure comme Il me paraît nécessaire de..., il pourrait répondre que C'est bien. C'est une bonne chose, sans produire d'action visant à répondre à la nécessité en question; quand on sait pourtant que la requête est un acte à visée pratique. La même chose pourrait se produire à cause d'un malentendu, involontaire et de bonne foi (malentendu envisagé ici comme un décalage interprétatif), suite à quoi la requête échouerait.

Toutefois, le contexte des Nations Unies, du moins celui de l'Assemblée Générale impose la bonne interprétation. L'indirection, même non conventionnelle, semble y être le code, tant et si bien qu'il paraîtrait saugrenu pour celui à qui on s'adresse de faire semblant de ne pas saisir le sens des requêtes. Il n'y a même pas de possibilité de malentendu, étant donné que les requêtes sont (jusqu'ici) exclusivement formulées de manière indirecte. L'indirection est donc une norme que les locuteurs maitrisent. Elle fait partie de ce qui construit la façon de parler à l'Onu et n'autorise pas d'interprétation erronée. En ces points, le discours diplomatique ou plutôt le discours «Onu» s'éloigne du fonctionnement du langage ordinaire. 
En résumé, à cause du flou qui est constamment entretenu sur leur contenu pragmatique et de la liberté interprétative qu'elles octroient en règle générale, les formulations indirectes, quelles qu'elles soient et à des degrés différents, sont toujours susceptibles d'être soumises à négociation ${ }^{15}$ entre les interlocuteurs. Il y a toujours une possibilité de discussion sur leur valeur de requête. Toutefois, cela ne s'applique pas nécessairement au genre des discours à l'Onu, dans lequel l'indirection est un code maitrisé et largement intégré dans les pratiques langagières.

\section{Répondre à l'exigence de politesse :}

37 La formulation indirecte des requêtes présente tout de même l'inconvénient d'être parfois complexe et aussi d'enfreindre le principe d'économie de la langue ${ }^{16}$, néanmoins les locuteurs s'y tiennent.

Le modèle de Brown et Levinson ou la théorie de la préservation des Faces ${ }^{17}$ postule que tout individu ressent le besoin de préserver sa face négative et sa face positive; celles-ci étant respectivement entendues comme le territoire corporel, matériel, spatial, temporel ou mental, et la face c'est-à-dire l'estime de soi, la bonne figure ${ }^{18}$. Cependant, beaucoup d'actes de langage parmi les plus courants sont potentiellement "menaçants " pour lesdites faces. C'est le cas de la requête par exemple, qui menace nécessairement la face négative de son destinataire; et qui, selon la manière dont elle est formulée peut aussi fortement porter atteinte à sa face positive. Par conséquent, pour atténuer ce caractère menaçant qui est inhérent à ce qu'on appelle les Face Threatening Acts (actes menaçants), l'on utilise la Politesse, en tant que «moyen de concilier le désir mutuel de préservation des faces, avec le fait que la plupart des actes de langage produits au cours de l'interaction sont potentiellement menaçants pour telle ou telle de ces mêmes faces ${ }^{19}$. La formulation indirecte des actes de langage fait ainsi partie des stratégies mises au service de l'exercice de la politesse.

Sachant que la requête quelle qu'elle soit est un acte hautement intrusif (en termes de face), les locuteurs prennent des précautions pour la construire : d'où l'indirection. Cette indirection répond à l'exigence de politesse qui est résolument une caractéristique importante du genre des discours prononcés à l'Onu, toujours dans l'objectif de réduire les risques de confrontation et de valoriser le consensus. En discours, l'indirection est concrètement initiée par l'usage de la troisième personne (le nom de pays), qui permet le transfert de l'autorité et de la responsabilité de l'énonciation, de la personne du représentant vers l'État-membre. Elle utilise ensuite des procédés conventionnels que la langue sait identifier comme porteurs de requête, ou bien des procédés non conventionnels qui nécessitent une interprétation qui tient compte du contexte énonciatif. À cette indirection s'ajoute souvent d'autres procédés tels que les nominalisations et les voix non actives.

40 Toutefois, l'on retrouve dans les discours aux Nations Unies une autre façon de formuler les requêtes:

\section{c. Les « tournures performatives »}

41 En dehors des requêtes indirectes conventionnelles et non conventionnelles, les actes de requête du corpus se réalisent fréquemment par l'usage de performatifs. Il s'agit de 
verbes qui ont pour caractéristique de dénommer l'acte qu'ils accomplissent en même temps qu'ils l'accomplissent.

Soient les énoncés :

(20) C'est par attachement à la Charte des Nations Unies que la Belgique, l'un des pays fondateurs, plaide avec insistance pour plus de rigueur et plus de transparence dans le fonctionnement et le budget de l'ONU, afin d'utiliser au mieux les moyens limités.

(21) La Belgique plaide également pour la résolution des causes profondes de l'instabilité dans cette région des Grands Lacs.

Le représentant de la Belgique exprime dans ces deux cas des requêtes, qui consistent à dire de son pays que celui-ci plaide pour quelque chose. Grâce à l'usage d'un performatif, il accomplit ce qu'il dit faire par le simple fait de le dire, il performe. En d'autres termes, le fait pour lui de dire que son pays plaide pour... accomplit l'action de plaider. En principe, « plaider pour » ne peut être performatif qu'à la première personne, c'est-à-dire que pour accomplir l'action de plaider par le simple fait de dire qu'on plaide, il faudrait que le je soit sujet, que ce soit le je lui-même qui plaide (je plaide). Ici, ce qui parait un constat à la troisième personne suffit pour accomplir l'action. Le représentant dit de son pays qu'il plaide, et l'action de plaider se réalise effectivement. Cependant, tel que nous l'avons souligné précédemment, le performatif se réalise car en réalité il ne s'agit plus ici d'une description. L'État se superpose à la parole du représentant, et la troisième personne devient une première personne. Étant donné le contexte institutionnel dans lequel la parole est prise et le rôle central de celui qui dit plaider (respectivement l'enceinte de l'Onu et la Belgique), l'action de plaider s'opère automatiquement et sans délai. Le fait que la première personne se substitue à la troisième et qu'en cela les performatifs se réalisent montre davantage l'impact d'un contexte socio-discursif sur le fonctionnement de l'énonciation.

Une multitude de verbes de la même sorte sont utilisés dans le corpus, afin de construire ce que nous appelons des requêtes « à tournure performative ». Des exemples en sont donnés ci-dessous :

(22) Face à de pareilles souffrances, les efforts et les progrès réalisés demeurent insuffisants. C'est pourquoi la Belgique insiste pour que puisse être établie sans délai une commission d'enquête internationale (pour la crise au Darfour).

(23) S'agissant de l'embargo imposé à la Grande Jamahiriya Arabe Libyenne Populaire et Socialiste, (...) Le Tchad demande non seulement la levée de cet embargo, mais est aussi opposé à toute nouvelle initiative unilatérale.

(24) Le G20 manque de légitimité et ne dispose pas des procédures transparentes pour décider des sanctions. (...) La Suisse préconise une égalité de traitement et une meilleure consultation entre les non-membres du G20.

(25) À l'échelle mondiale, les coûts des adaptations au changement climatique devraient atteindre plusieurs dizaines de milliards de dollars, dont plus de la moitié à la charge des pays en développement. La Suisse propose la création d'une taxe $\mathrm{CO} 2$ à l'échelle mondiale fondée sur le principe du pollueur-payeur afin de faire face à ces nouveaux besoins.

(26) Concernant les crises dans le monde, (...) la République Démocratique du Congo recommande, plus que jamais, leur règlement par la voie pacifique, dans le respect de la Charte des Nations Unies et des résolutions pertinentes du Conseil de Sécurité. (27) La RCA qui a souscrit à la déclaration du Millénaire exhorte les pays du Nord à déterminer avec l'Afrique un partenariat réel, tant public que privé, qui puisse concourir à appuyer les efforts de développement de ce continent.

(28) Les situations en Afghanistan et en Iraq demeurent préoccupantes. La République Démocratique du Congo engage la Communauté internationale à 
l'éradication du fléau du terrorisme afin de permettre à ces deux pays de reprendre pleinement la place qui est la leur au sein de l'échiquier international.

(29) C'est en vertu de ces valeurs que le gouvernement gabonais a décidé d'abolir la peine de mort. Le Gabon invite les États membres à témoigner leur appui au projet de résolution sur l'instauration d'un moratoire sur la peine de mort dont il est coauteur.

(30) Le Gabon appelle à la destruction de tous les arsenaux chimiques dans le monde.

Ainsi, pour exprimer une requête aux Nations Unies, très souvent, le pays-locuteur plaide, sollicite, invite à, se joint à l'invitation d'un autre, propose, soutient ou s'associe à la proposition d'un autre, il demande, insiste, encourage, suggère, exhorte, recommande, engage les autres à, il prône, préconise, formule/forme le veeu de et appelle à (avec toutes ses variantes dont renouveler son appel/lancer un appel/réitérer l'appel lancé/joindre sa voix à l'appel lancé/appeler de ses voux...).

Tous ces verbes ont en réalité vocation à construire des actes directs, puisqu'ils viennent préciser clairement «le statut pragmatique de l'énoncé » (Kerbrat-Orecchioni, 2001, p.36). Autrement dit, ils disent explicitement que la requête est une recommandation, une invitation, un encouragement, une exhortation... Ils permettent ainsi d'échapper à toute forme d'ambiguïté interprétative. Néanmoins, si les valeurs exprimées performativement sont en principe « in-contestables, in-déniables et non-négociables » (Kerbrat-Orecchioni, 2001, p.48), et qu'elles semblent donc moins indirectes, dans notre étude, ces formulations continuent sous d'autres aspects à répondre au principe de politesse, de par les faits de langue qui s'y appliquent. Il s'agit -tel que relevé précédemment- des procédés de nominalisations et d'usage des voix que l'on y retrouve, surtout lorsque des questions épineuses sont traitées. Ainsi, à qui le Tchad (23) demande-t-il « la levée de l'embargo » contre la Libye ? Qui est-ce qui a « imposé » cet embargo ? Et qui serait à l'origine de l'« initiative unilatérale » à laquelle il s'oppose ? Et au sujet du manque de transparence du G20 selon la Suisse (24), qui est-ce qui doit mieux traiter et consulter les non-membres du G20?

\section{Un mode de prise de parole influencé par le contexte}

Il faut noter que la politesse en tant que norme pour le genre des discours aux Nations vise à réduire les conflictualités et à valoriser le consensus (au moins en discours), ce qui correspond à l'identité de l'institution. Cependant, elle est aussi imposée par le cadre juridique qui régit les prises de parole. Par exemple, les demandes que l'on exprime de manière performative ne sauraient être des ordres, ni des injonctions en ceci que les décisions à prendre sont collectives (en principe), passent nécessairement par un vote général et concernent l'intérêt commun. Pour les procédés secondaires qui renforcent souvent l'exercice de la politesse, la Suisse par exemple ne peut " proposer » à personne en particulier «la création d'une taxe $\mathrm{CO} 2$ à l'échelle mondiale», puisque c'est une décision qui concerne toute la communauté, et qui pour être prise passe par l'observation de plusieurs codes et règles juridiques. Il en va de même lorsque le Gabon «appelle à la destruction" de tous les arsenaux chimiques dans le monde. Dès lors, la politesse, manifestée par toutes les formes linguistiques que nous avons recensées, est donc une contrainte qui est liée non seulement à la diplomatie mais aussi à la conjoncture réglementaire dans laquelle les discussions évoluent. 
Notre étude, nécessairement soumise à la restriction à un corpus bien délimité, s'est focalisée exclusivement sur le discours à l'Onu. Ainsi, même en considérant que les discours à l'Onu ne méritent pas de former à eux-seuls un sous-genre en ce qu'ils ne se distinguent en rien d'autres discours qui évoluent dans le même type de contexte, nous ne saurions toujours pas avoir la prétention d'étendre le constat de la singularité de leur fonctionnement pragmatico-énonciatif (s'il est valide) à tous les discours qui répondent aux mêmes contraintes réglementaires et diplomatico-institutionnelles que les discours à l'Onu. C'est pourquoi nous nous limiterons à répéter que : sous l'aspect unique (pour l'instant) des actes de langage, le discours à l'Onu s'éloigne du fonctionnement de l'énonciation dans le langage ordinaire.

\section{BIBLIOGRAPHY}

ADAM J. M., 1997, «Genres, textes, discours : Pour une reconception linguistique du concept de genre », Persée, Langues et littératures modernes, pp. 665-681.

ADEGBOKU D., 2011, « De l'économie en langue ou dans le langage : une linguistique 'des temps qui pressent' ", Synergies Afrique Centrale et de l'Ouest nº, pp. 25-37.

AUSTIN J., 1970, Quand dire c'est faire, Paris, Le Seuil « Points ».

AMOSSY R., 2000, L'argumentation dans le discours, Paris, Nathan.

AMOSSY R., 1999, Images de soi dans le discours. La construction de l'Ethos, Lausanne-Paris, Delachaux et Niestlé S.A.

ANSCOMBRE J-C. et DUCROT O., 1988, L'argumentation dans la langue, Liège, Mardaga.

ARISTOTE, 1991, Rhétorique, Introduction de Michel Meyer, Paris, Le Livre de poche. 
BONHOMME M., 2013, « Argumentation et interaction dans les brochures du Conseil fédéral suisse sur les votations populaires », Argumentation et Analyse du Discours [En ligne], mis en ligne le 10 avril 2013.

BOURDIEU P., 1982, Ce que parler veut dire. L'économie des échanges linguistiques, Paris, Fayard.

BROWN P. \& LEVINSON S. C., 1987, Politeness: Some Universals in Language Usage, Vol. 4, Cambridge University Press, Cambridge.

CHARAUDEAU P. \& MAINGUENEAU D. (éd.), 2002, Dictionnaire d'analyse du discours, Paris, Le Seuil.

COHEN-Wiesenfeld S., 2004, «L'inscription de la subjectivité dans le discours diplomatique », Semen, no 17, pp. 41-58.

COZMA A., 2012, «Fondements sémantiques et réalisations linguistiques de l'acte de langage reprocher ", Signes, Discours et Sociétés, [en ligne], 9. La force des mots : les mécanismes sémantiques de production et l'interprétation des actes de parole « menaçants ».

DANBLON E., 2010, «À quelles conditions une charte est-elle efficace ? ", Argumentation et Analyse du Discours [En ligne], mis en ligne le 15 avril 2010.

DE FORNEL M., 1983, «Légitimité et actes de langage », Actes de la recherche en Sciences Sociales, $\mathrm{n}^{\circ} 1$, pp. $31-38$.

DELBECQUE N., 2002, Linguistique cognitive, Comprendre comment fonctionne le langage, De Boeck-Duculot, Bruxelles.

GOFFMAN E., 1973, La mise en scène de la vie quotidienne, 2. Les relations en public, Paris, Minuit.

GOFFMAN E., 1974, Les Rites d'interaction, Paris, Minuit.

GOFFMAN E., 1987, Façons de parler, Paris, Minuit.

KERBRAT-ORECCHIONI C., 1980, L'énonciation. De la subjectivité dans le langage, Paris, Armand Colin.

KERBRAT-ORECCHIONI C., 2001, Les actes de langage dans le discours, Paris, Minuit.

KRIEG-PLANQUE A. \& OGER C., 2010, « Discours institutionnels. Perspectives pour les sciences de la communication », Mots : Les Langages du Politique, Trente ans d'étude des langages du politique, pp. 91-96.

Krieg-Planque A., 2013, Analyser les discours institutionnels, Paris, Armand Colin.

KRIEG-PLANQUE A., 2013, « Un discours sur prescription : les « argumentaires » des partis politiques comme éléments de cadrage de la parole », Argumentation et Analyse du Discours [En ligne], mis en ligne le 10 avril 2013.

LAUGIER S., 2005, « Actes de langage et état de choses : Austin et Reinach », Les Études philosophiques, $\mathrm{n}^{\circ} 72$, pp. 73-97.

MAINGUENEAU D., 2004, « Typologie des genres de discours », dans Le Discours littéraire. Paratopie et scène d'énonciation, Paris, Armand Colin.

MAINGUENEAU D., 2007, « Genres de discours et Modes de généricité », Le Français aujourd'hui, n ${ }^{\circ} 159$.

MAYAFFRE D., 2004, Paroles de présidents, Jacques Chirac (1995-2003) et le discours présidentiel sous la Ve République, Paris, Honoré Champion. 
MAYAFFRE D., 2012, Nicolas Sarkozy, Mesure et démesure du discours, 2007/2012, Paris, Presses de la Fondation Nationale des Sciences Politiques.

MAYAFFRE D., 2000, Le poids des mots, Le discours de gauche et de droite dans l'entre-deuxguerres, Paris, Honoré Champion.

MONTE M. et OGER C., 2015, « Discours d'autorité : Des discours sans éclat(s) ? », Mots. Les langages du politique, $n^{\circ} 107$.

RASTIER F. \& PINCEMIN B., 1999, « Des genres à l'intertexte », Cahiers de praxématique 33. Pulm. p. 83-111.

RASTIER F., 2001, «Éléments de théorie des genres ». Texto ! [en ligne], Disponible sur <http:// www.revue-texto.net/Inedits/Rastier/Rastier_Elements.html>.

ROSSARI C., 2013, «Phénomènes énonciatifs et argumentation dans les brochures du Conseil fédéral suisse sur les votations populaires ", Argumentation et Analyse du Discours [En ligne], mis en ligne le 10 avril 2013.

SEARLE J., 1972, Les actes de langage. Essai de philosophie linguistique, traduction par Hélène Pauchard, Paris, Hermann.

SEARLE J., 1982, Sens et expression, Paris, Minuit.

VILLAR C., 2006, Le discours diplomatique, Paris, L'Harmattan.

\section{NOTES}

1. Des termes sont également sous-utilisés, on parle alors de spécificité négative.

2. Cette restriction aux noms et adjectifs pour une première étude des spécificités est empruntée à Damon Mayaffre au chapitre 1 de Paroles de président, dans lequel il parle d'ailleurs de "mots pleins» pour désigner des termes quasi-systématiquement rencontrés dans les discours présidentiels français, et qui en forment finalement le «squelette lexical ». L'analyse globale comportant tous les codes grammaticaux y compris les signes de ponctuation étant trop large pour le propos de notre contribution, nous avons jugé plus pertinent de nous limiter pour un début aux substantifs et adjectifs.

3. Le nombre élevé d'occurrences des noms de pays dans la totalité du corpus atteste de l'importance de la prise en charge énonciative par les pays-locuteurs. Il varie entre 156 pour le Tchad) et 83 pour la Suisse, sachant que cette dernière n'a intégré les Nations Unies que dès l'année 2002.

4. KRIEG-PLANQUE A. (2013).

5. AUSTIN J. (1970).

6. Mickail Kissine discute la notion d'acte expressif, mais le propos de cet article est autre que la part expressive des discours aux Nations unies.

7. Voir KERBRAT-ORECCHIONI (2001) p.41 et SEARLE (1982) p.72.

8. Ceci n'exclut pas que les discours laissent souvent apparaître des marques d'hostilité, comme les exemples 1 et 2 le montrent. Mais il faudrait pour mieux s'y intéresser aborder les problématiques liées à l'implicite, qui ne sont pas encore étudiées dans cette partie, celle-ci s'intéressant à l'indirection en tant qu'elle contribue à caractériser « le genre » des discours à l'onu.

9. Il n'aurait pas été possible d'employer ces procédés avec l'impératif. «La France souhaite le retrait de Gaza » n'aurait pu donner de manière directe que Retirez-vous de Gaza ou encore Que vos troupes se retirent de Gaza. C'est la formulation «La France souhaite» qui permet 
d'employer le substantif et partant, de ne pas indexer celui à qui le message est destiné et aussi de sembler moins injonctif.

10. Voir aussi que dans l'énoncé tel qu'il a été posé dans le discours prononcé, c'est-à-dire La France souhaite que le retrait de Gaza en soit une première étape (au processus de paix agréé par le conseil de sécurité), le retrait de Gaza, n'est pas (en principe) l'objet du dire. C'est plutôt son rang parmi les étapes du processus de paix qui semble être mis en avant, et ledit retrait apparait alors comme déjà convenu.

11. DANBLON E. (2010).

12. KRIEG-PLANQUE A. \& OGER C. 2010, mis en ligne le 06 novembre 2012. Les auteures postulent dans cet article que les institutions, afin de gagner en légitimité, tendent à éliminer les conflictualités en discours au profit de la valorisation du consensus.

13. Ce sont les représentants eux-mêmes qui parlent, dans l'enceinte des Nations Unies, et non par exemple des journalistes dans un reportage.

14. Voir COZMA A. (2012).

15. Voir la Négociabilité dans KERBRAT-ORECCHIONI C. (2001).

16. Entendue ici comme une logique de réduction des dépenses langagières, pour arriver à dire beaucoup avec peu de productions langagières sonores ou écrites. Voir ADEGBOKU D. (2011) pp. 25-37.

17. La notion de Face a initialement été empruntée à GOFFMAN E. (1973).

18. BROWN P. \& LEVINSON S. C. (1987).

19. KERBRAT-ORECCHIONI C. (2001) p.17.

\section{ABSTRACTS}

The study of directive speech acts in United Nations speeches has proved very useful in analyzing and classifying the diplomatic sub-genre in discourse. The indirect formulation of speech acts and the use of various processes in order to avoid potential risks of conflict reveal the "politeness " constraint met by these speeches, in compliance with a principle of promotion of consensus as an institutional duty. These speeches in which the utterance act responsibility lies with the member states - as they are authentic locutors speaking on their own behalf - are at variance with ordinary language. It is possible to observe the influence of the social discursive context (diplomatic and legal) on language use. Speech acts of requesting in these speeches point out member states' concerns, since a request can be thought of as an indicator of lack. The repeated use of specific illocutionary verbs gives a good overview of language practices becoming systematic.

Étudier les actes de requête dans les discours prononcés aux Nations Unies s'avère d'une utilité certaine dans le travail de caractérisation de ce sous-genre diplomatique. La formulation indirecte des actes de langage et les procédés linguistiques qui éloignent les sources potentielles de confrontation révèle des discours contraints par l'exercice de la "politesse ", au service de l'impératif institutionnel de valorisation du consensus. Ces discours qui s'éloignent du fonctionnement du langage ordinaire transfèrent la prise en charge de l'énonciation aux Étatsmembres qui sont ici de véritables locuteurs et qui partant parlent en leur propre nom. L'on observe alors l'impact d'un contexte socio-discursif (cadre diplomatique et juridique) sur le fonctionnement de langue. Les requêtes formulées dans ces discours reflètent les problématiques 
qui préoccupent les États membres, puisque dans bien des cas, une requête peut être l'aveu d'un manque. Les verbes illocutoires donnent un bon aperçu des «façons de parler » et témoignent d'ores-et-déjà d'une routinisation des pratiques langagières.

INDEX

Mots-clés: énonciation, actes de langage, politesse, genre, Nations Unies

Keywords: utterance production, speech acts, politeness, genre, United Nations

\section{AUTHOR}

OLIVIA DE MATTEI TCHEMAKO

BABEL EA 2649 - Université de Toulon 